\title{
Modelling and Design of Polygon-Shaped Kinesin Substrates for Molecular Communication
}

\author{
Nariman Farsad ${ }^{1}$, Andrew W. Eckford ${ }^{1}$, and Satoshi Hiyama ${ }^{2}$ \\ ${ }^{1}$ Dept. of Computer Science and Engineering, York University, Toronto, Canada M3J 1P3 \\ ${ }^{2}$ Research Laboratories, NTT DOCOMO Inc., Yokosuka, Kanagawa, Japan \\ Email: nariman@cse.yorku.ca, aeckford@yorku.ca, hiyama@nttdocomo.co.jp
}

\begin{abstract}
One of the most prominent forms of information transmission between nano- or micro-scale devices is molecular communication, where molecules are used to transfer information inside a fluidic channel. The effects of channel shape on achievable information transmission rates is considered in this work. Specifically, regular convex polygons are studied. A mathematical framework for finding the optimal channel among this class of geometric shapes is derived. Using this framework it is shown that the optimal channel tends to be circular. This result is verified using computer simulations.
\end{abstract}

Index Terms-Molecular Communication, Information Theory, Simulation, Design.

\section{INTRODUCTION}

With the prevalence of nanotechnology, creation of nanoscale devices such as nanorobots is close to reality. Typically a nanodevice can perform a specific and simple task. However, to accomplish complex goals, a network of nanodevices must be employed. Traditional communication schemes cannot be directly applied at nano-scales. To overcome this obstacle, a new and emerging field of nanoscience called nanonetworks [1], [2] is proposed, where nano- or micro-scale devices communicate over small distances typically in the order of a few hundred nanometers or a few hundred micrometers.

One of the most prominent modes of communication at nano-scales is molecular communication [2], which is a biologically-inspired communication technique where nanoscale particles such as molecules are used to convey information. Information can be conveyed through encoding messages into the release timing [3], number [4], [5], concentration [6], or identities of particles [7], which are released by a transmitter and propagate to a receiver.

One important practical domain for molecular communication is in microfluidics, where chemical processes are carried out in very small engineered aqueous channels. There are many potential applications for this domain, such as lab-onchip devices [8], and point-of-care diagnostic chips [9]. Although microfluidic systems are better described as microscale systems, their fundamental components (e.g. molecular motors, microtubules and information particles) have nanoscale dimensions. For example, the width of a microtubule (MT) filament is around 25 nanometers.

Two different propagation schemes can be employed in aqueous solutions: passive transport, and active transport. In passive transport, the information-carrying particles propagate from the transmitter to the receiver by diffusing in the mircofluidic medium without using external energy. In active transport, information-carrying particles are transported by an external means such as molecular motors or an external device such as a syringe pump.

Besides regular diffusion based molecular communication proposed in [10], passive transport through gap junction channels is also proposed in [11]. Different active transport propagations are also possible. In [12], kinesin molecular motors moving over immobilized MT tracks are proposed for carrying information particles. One disadvantage of this scheme is the challenge associated with alignment of polarity of the MT tracks. To overcome this issue, a system composed of MT filaments moving over kinesin covered substrate is proposed in [13]. Bacterial based communication has also been proposed in [7], [14], [15], where flagella propelled microbes are proposed for transferring the information particles. However, one of the issues with this propagation scheme is the lack of experimental demonstration of working system components.

In [5], [16], it was shown that active transport can achieve higher information rates than passive transport in confined microfluidic channels. Therefore, in this work, we consider active transport using MT filaments gliding over kinesin covered substrate. In previous works, different design optimizations have been performed based on this particular propagation scheme. Specifically, in [17] the shape of the transmission area is optimized, while in [4] the size of the vesicles encapsulating the information particles is optimized.

One of the open problems in designing microfluidic channels is designing the shape of the microchannel itself. Previous works [18] have demonstrated that the shape of the channel has significant effect on the rate of information transfer. In particular, in [19] it is shown that when rectangular channels are considered, the square-shaped channels have the highest information rate.

In this paper we extend our previous work with rectangular channels, and consider a larger class of channel shapes. Specifically, regular convex polygons are considered in this work. Our main contributions include: devising a mathematical framework for finding the optimal channel shape for this class, and performance evaluation of this framework through a devised simulation environment. Based on the obtained results we show that circular-shaped channels tend to be the optimal channel geometry, and achieve the highest information rates. 


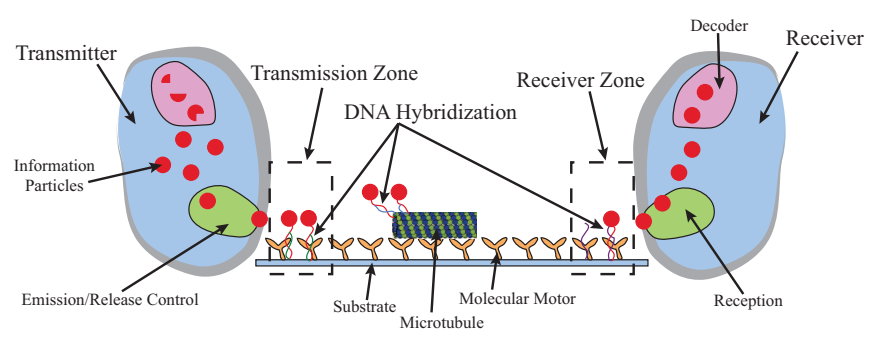

Fig. 1. Depiction of the communication environment.

This increase in achievable information rates could potentially be translated into lab-on-chip and diagnostic chip devices that produce the end results faster.

\section{Active Transport Molecular Communication ENVIRONMENT}

Our molecular communication environment is similar to the ones in [13], [16], [20], where MTs gliding over kinesin covered substrate are used to transfer the information particles from a transmitter to a receiver. The propagation environment is considered to be a regular polygon with a transmission zone and a receiver zone. Regular convex polygons, are equiangular (all angles are equal in measure) and equilateral (all sides have the same length). This class of polygons includes geometric shapes, ranging from an equilateral triangle, square, pentagon, and hexagon, all the way up to a circle as number of sides approaches infinity. Message-bearing particles originate at the transmission zone. These particles are initially assumed to be anchored to the transmission zone until MT filaments, moving over molecular motor tracks that cover the whole environment, pick up and transport the information-carrying particles from the transmission zone to the receiver zone. As in [13], we assume deoxyribonucleic acid (DNA) hybridization bonds are used to anchor, load and unload the information particles. This process is summarized in Figure 1 and the reader is referred to [13] for detailed explanation.

We use Monte-Carlo techniques proposed in [21] to simulate the motion of the MT. We also use the grid loading mechanism proposed in [4], to simulate the information particles' loading and unloading. For loading an information particle, the MT filament must drive close to the anchored particle. Therefore, we divide the transmission zone into a square grid, where the length of each square in the grid is the same as the diameter of the particles. We then distribute particles randomly and uniformly among the squares in the grid. In general, we assume that the MTs can load multiple particles, which we know to be possible based on lab experiments; thus, if a MT enters a square which is occupied by a particle, and it has an empty loading slot available, we assume the MT loads that particle. For unloading, we assume all the loaded particles are unloaded as soon as a MT enters the receiver zone.

In [4], it was shown that the optimal transmission zone is along the walls of the channel since MTs mostly glide very close to channel walls. This positioning increases the chance

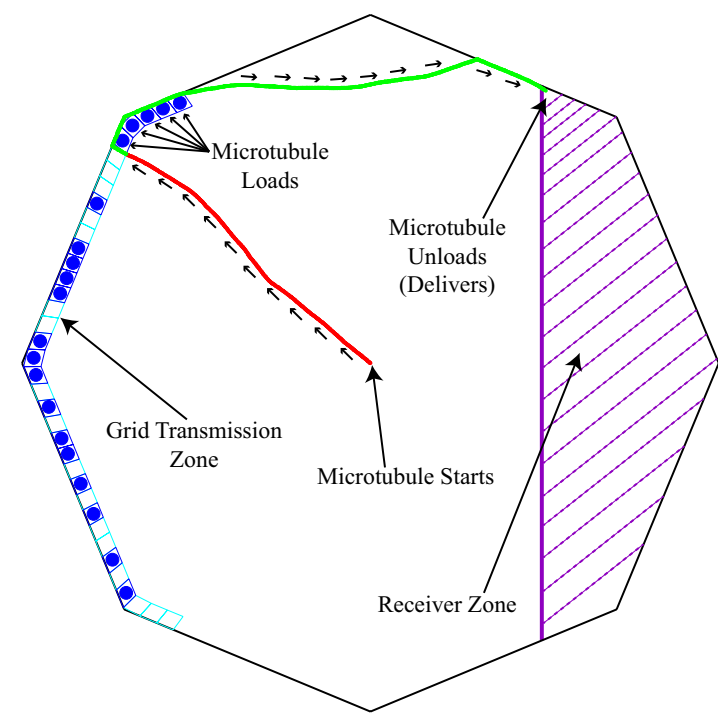

Fig. 2. Depiction of the simulation environment.

of a MT picking up an information particle during its trips. Therefore, in this work we always assume the grid loading structure is along the walls of the channel regardless of the channel shape.

Figure 2 shows our simulation environment, where a MT starts from the center of an octagon-shaped channel (8-sided regular polygon), and moves to the grid transmission structure on the left. The MT then picks up five information particles (in the figure, the color of the MT trajectory turns green after the first particle is loaded), and continues its path to the right until the picked up particles are unloaded at the receiver zone.

\section{Channel Shape Analysis}

The shape of the kinesin covered channel has a significant effect on channel capacity as shown in [18], [19]. In this work, we optimize the channel shape with respect to the channel capacity. As channel capacity increases, the maximum rate of information transmission increases. Maximizing channel capacity has been one of the important areas of research in traditional communication systems. For molecular communication channels, this potentially translates to faster and more efficient diagnostic results in applications such as point-of-care and lab-on-chip devices.

\section{A. Channel Capacity}

In this work, we assume information is conveyed through mass transfer. In the simplest possible conception of this scheme, the particles themselves are not information-bearing, and a message is conveyed in the number of particles released by the transmitter. Let $X \in \mathcal{X}=\left\{0,1,2, \cdots, x_{\max }\right\}$ represent the number of information particles released into the medium by the transmitter, and $x_{\max }$ be the maximum number of particles the transmitter can release per channel use. Each value of $X$ represents a different message. However, this 
message might not be perfectly conveyed to the receiver: given a time limit $T$ for the communication session, it is possible that some of the particles will not arrive at the receiver after $T$ has elapsed. We refer to time limit $T$ as time per channel use. In other words, time per channel use is a predefined amount of time representing the time duration for a single message transmission session, and it is one of the parameters of the molecular communication system.

Let $Y \in \mathcal{X}$ represent the number of information particles that arrive at the destination after the time per channel use interval $T$. The maximum rate at which any communication system can reliably transmit information over a noisy channel is bounded by a limit called channel capacity. The channel capacity can be calculated as,

$$
C=\max _{f_{X}(x)} I(X ; Y),
$$

where $I(X ; Y)$ is the mutual information between $X$ and $Y$. Mutual information is defined as

$$
I(X ; Y)=E\left[\log _{2} \frac{f_{Y \mid X}(y \mid x)}{\sum_{x} f_{Y \mid X}(y \mid x) f_{X}(x)}\right],
$$

where, $f_{Y \mid X}(y \mid x)$ represents the probability of receiving symbol $y$ at the destination, given that symbol $x$ was transmitted by the source; $f_{X}(x)$ represents the probability of transmitting symbol $x$; and $E[\cdot]$ represents expectation.

\section{B. Average Number of MT Trips}

One of the most important factors affecting the channel capacity is the number of MT trips, $K$, during a single time per channel use interval $T$. We define a single $M T$ trip as the movement of the MT from anywhere in the channel to the transmission zone and then the receiver zone. For example, a single MT trip is shown in Figure 2. After the MT completes its first trip, subsequent trips are defined as the movement of the MT from the receiver zone to the transmission zone and back. During any trip, a MT can deliver 0 or more information particles (up to its maximum load capacity). Because the motion of the MT filaments are random in nature, for a given value of time per channel use $T$, the number of trips is random. In the rest of this section, we derive an estimated closed-form expression of the average number of MT trips.

Let the random variable $S^{(T)}$ be the number of trips for a single MT during the time per channel use interval, $T$. Let $v_{\text {avg }}$ be the average speed of the MTs, $r$ be the radius of the regular polygon, and $n$ be the number of sides. A good estimate for the average number of MT trips, when a single MT is employed is given by,

$$
E\left[S^{(T)}\right] \approx \frac{v_{\mathrm{avg} T}}{P(r, n)},
$$

where $P(r, n)$ is the perimeter of the regular polygon, given by,

$$
P(r, n)=2 n r \sin (\pi / n) .
$$

This means on average from each trip, the MT travels a distance equivalent to the perimeter of the channel. We use this

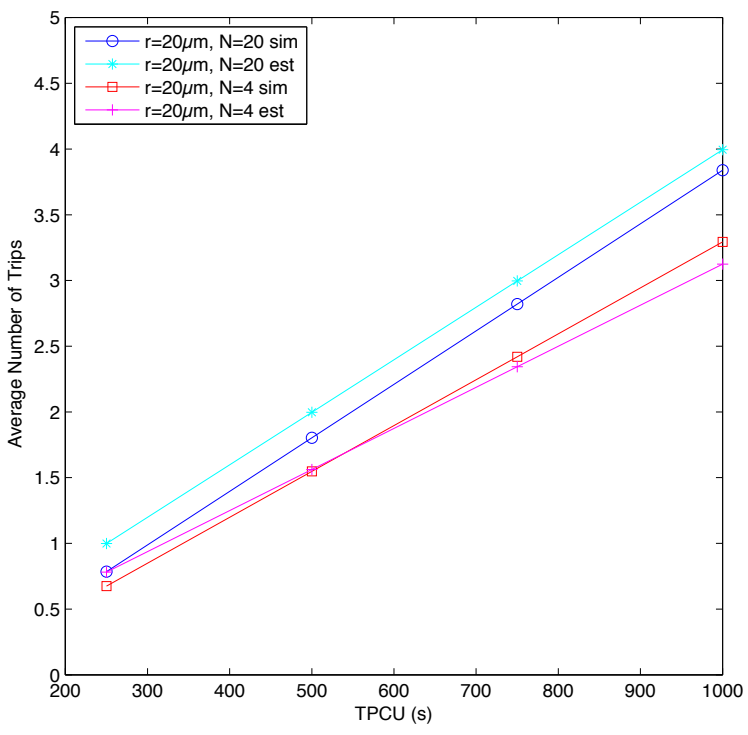

Fig. 3. Average number of trips.

estimate because MTs usually follow the walls of the channel. Figure 3 shows the difference between the estimated average obtained from Equation (3), and the simulated.

In practice, there are typically more than one MT inside the channel. Furthermore, the number of MTs is not a constant, but is dependent on the volume of fluid in the channel: the DNA-covered MTs are prepared in chemical solutions, and therefore have a constant concentration inside the solution. Let $C$ be the concentration of MTs as number of MTs per unit volume, and $h$ be the height of the channel. If we assume $C$ and $h$ are constants, then the number of MTs inside the channel is given by

$$
M=A(r, n) \times h \times C,
$$

where $M$ is the number of MTs, $A(r, n)$ is the cross sectional area of the channel calculated as

$$
A(r, n)=0.5 n r^{2} \sin (2 \pi / n),
$$

for regular polygons.

Let the random variables $K_{1}^{(T)}, K_{2}^{(T)}, K_{3}^{(T)}, \ldots, K_{M}^{(T)}$ be the number of trips for each of the $M$ MTs during $T$ seconds. The total number of MT trips during $T$ seconds is therefore given by,

$$
K^{(T)}=\sum_{i=1}^{M} K_{i}^{(T)} .
$$

The average number of MT trips during $T$ seconds is therefore calculated as,

$$
E\left[K^{(T)}\right]=\sum_{i=1}^{M} E\left[K_{i}^{(T)}\right] .
$$

We assume that the number of trips for individual MTs are independent and identically distributed, because they are 
chemically similar and don't interact with each other. Due to the identical distributions, the equation simplifies to

$$
E\left[K^{(T)}\right]=\sum_{i=1}^{M} E\left[S^{(T)}\right]=M E\left[S^{(T)}\right] .
$$

Using the approximation shown in Equation (3), and Equation (5), the total number of trips can be estimated as

$$
E\left[K^{(T)}\right] \approx T v_{\text {avg }} C h \frac{A(r, n)}{P(r, n)},
$$

where $A(r, n)$ and $P(r, n)$ are the cross sectional area and perimeter of the channel, respectively.

Substituting Equations (4) and (6) into Equation (10) and simplifying we get,

$$
E\left[K^{(T)}\right] \approx 0.5 T v_{\mathrm{avg}} C h r \cos (\pi / n),
$$

where we have used the fact that $\sin (2 u)=2 \sin (u) \cos (u)$.

\section{Optimal Channel Design}

Based on the closed-form expression derived in the previous section, a number of different design strategies can be outlined. These strategies are based on the assumption that maximizing the average number of MT trips, maximizes the channel capacity. Although this assumption may hold true, it must be noted that the variance of the number of MT trips effects channel capacity as well. However, deriving an estimated closed-form expression for the variance of the number of MT trips is generally more difficult.

1) Constant Radius: If we assume the channel radius is a constant, then from Equation (11) it is evident that average number of MT trips is maximized, if $\cos (\pi / n)$ is maximized. Because $n$ is the number of polygon sides, and it maximizes the average number of MT trips as it approaches infinity, the optimal channel shape in this case is a circular-shaped channel.

2) Constant Area: If we assume the cross sectional area of the channel is a constant, then from Equation (10) it is evident that the shape with the smallest perimeter would maximize the average number of MT trips. Under this condition again the circular-shaped channel is the optimal shape because it has the largest ratio of area to perimeter among regular polygons with constant area.

3) Constant Perimeter: If we assume the perimeter of the channel is a constant, then from Equation (10) it is evident that the shape with the largest area would maximize the average number of MT trips. Circular-shaped channel is again the optimal shape because it has the largest ratio of area to perimeter among regular polygons with constant perimeter.

\section{RESUlts}

To verify the optimal channel design strategies we developed based on the derived closed-form expression in the previous section, we use computer simulations. These simulations are performed in the simulation environment similar to the one developed in [4]. The parameters used in the simulations are as follows: simulation time steps of $\Delta T=0.1$ seconds, MT diffusion coefficient $D=2.0 \cdot 10^{-3} \mu^{2} / \mathrm{s}$, average speed

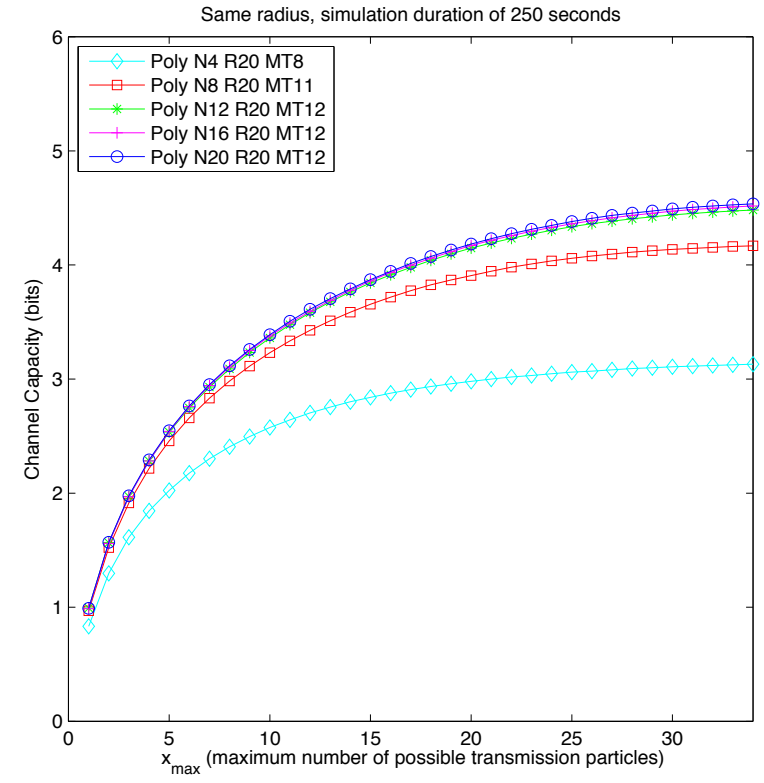

Fig. 4. Channel capacity in bits versus $x_{\max }$, maximum number of information particles that can be released by the transmitter.

of the MT $v_{\text {avg }}=0.5 \mu \mathrm{m} / \mathrm{s}$, and persistence length of the MT trajectory $L_{p}=111 \mu \mathrm{m}$. We also assume the size of the information particles is $1 \mu \mathrm{m}$, the average length of the MTs is $10 \mu \mathrm{m}$, and each MT can load up to 5 information particles in one trip from the transmission zone to the receiver zone. When multiple MTs are used, we assume the concentration of MTs is constant at $C=0.001 \mathrm{MT} / \mathrm{fL}$, and the height of the channel is always constant at $h=10 \mu \mathrm{m}$ regardless of the channel shape. These parameters are all selected based on experimental observations of DNA covered MTs moving over a kinesin covered substrate.

We first consider channels with constant radius $r=20$ $\mu \mathrm{m}$ but different number of sides $n$. Figure 4 shows the results for time per channel use interval of 250 seconds. The number of MTs inside each channel is an integer. Therefore, we use the floor function $\lfloor$.$\rfloor on Equation (5) to get \lfloor M\rfloor$. From the figure it is evident that as the number of sides increases the channel capacity increases. Therefore, as the channel becomes more circular, the channel capacity increases. This result supports the design criteria we developed using the closed-form expression in the previous section.

Channels with constant cross sectional area are considered next. In this case because the height of the channels are also assumed to be constant, the number of MTs inside each channel is the same. Therefore, we simulate each channel with a single MT for simplicity. We consider channels with constant cross sectional area $A=1600 \mu \mathrm{m}^{2}$, and time per channel use interval of 1000 seconds in Figure 5. We can see that as channel becomes more circular (i.e. number of sides increases), the channel capacity increases. This is in agreement with our proposed design strategy.

Channels with constant perimeter are considered last. In 


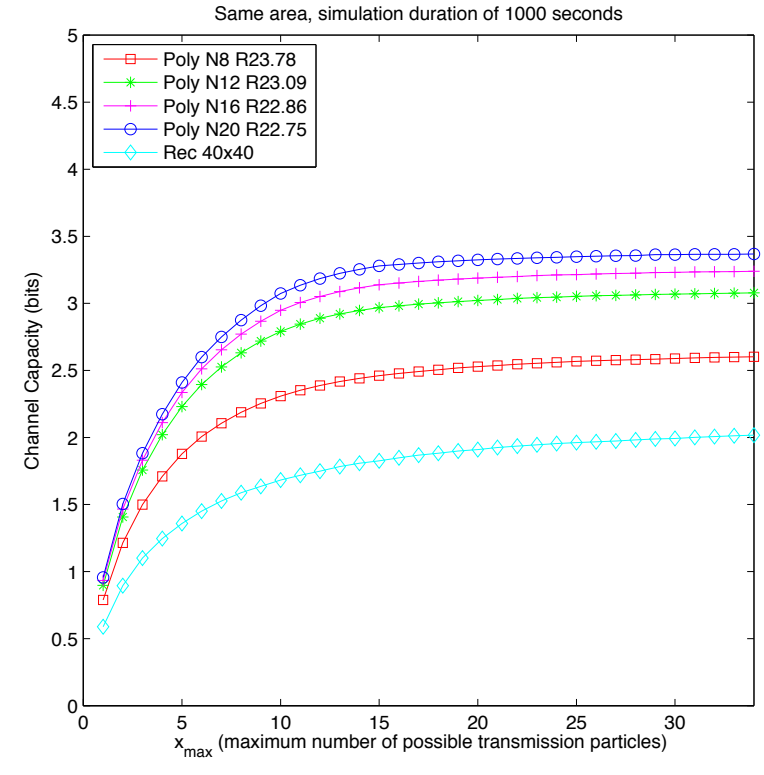

Fig. 5. Channel capacity in bits versus $x_{\max }$, maximum number of information particles that can be released by the transmitter.

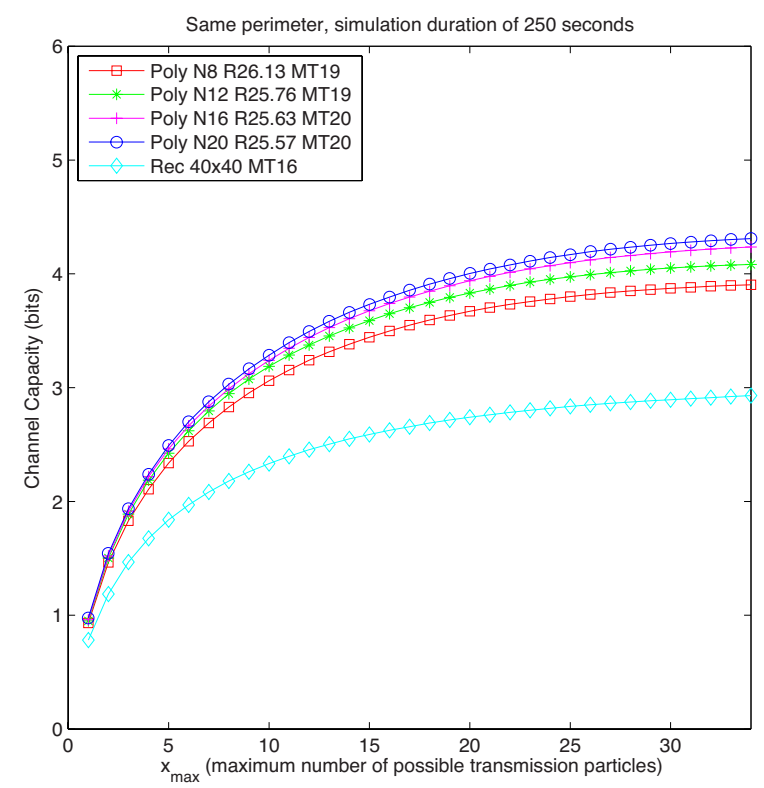

Fig. 6. Channel capacity in bits versus $x_{\max }$, maximum number of information particles that can be released by the transmitter.

particular, channels with perimeter $P=160 \mu \mathrm{m}$, and time per channel use interval of 250 seconds are considered for different number of sides $n$. Figure 6 shows the results. Again it is evident that as channel becomes more circular, the channel capacity increases. Based of these results and the results obtained from the estimated closed-form expression we conclude that circular-shaped channels tend to be the optimal choice for kinesin covered molecular communication channels.

\section{REFERENCES}

[1] I. F. Akyildiz, F. Brunetti, and C. Blazquez, "Nanonetworks: A new communication paradigm," Computer Networks, vol. 52, no. 12, pp. 2260 2279, 2008.

[2] S. Hiyama and Y. Moritani, "Molecular communication: harnessing biochemical materials to engineer biomimetic communication systems," Nano Communication Networks, vol. 1, no. 1, pp. 20-30, 2010

[3] A. W. Eckford, "Timing information rates for active transport molecular communication," in Nano-Net, vol. 20 of Lecture Notes of the Institute for Computer Sciences, Social Informatics and Telecommunications Engineering, pp. 24-28, Springer Berlin Heidelberg, 2009.

[4] N. Farsad, A. W. Eckford, S. Hiyama, and Y. Moritani, "Quick system design of vesicle-based active transport molecular communication by using a simple transport model," Nano Communication Networks, vol. 2, no. 4, pp. 175-188, 2011.

[5] N. Farsad, A. Eckford, S. Hiyama, and Y. Moritani, "On-Chip Molecular Communication: Analysis and Design," IEEE Transactions on NanoBioscience (in press), 2012.

[6] M. U. Mahfuz, D. Makrakis, and H. T. Mouftah, "On the characterization of binary concentration-encoded molecular communication in nanonetworks," Nano Communication Networks, vol. 1, no. 4, pp. 289-300, 2010.

[7] L. C. Cobo and I. F. Akyildiz, "Bacteria-based communication in nanonetworks," Nano Communication Networks, vol. 1, no. 4, pp. 244 256, 2010.

[8] R. Daw and J. Finkelstein, "Insight: Lab on a chip," Nature, vol. 442, no. 7101, pp. 367-418, 2006.

[9] P. Yager, T. Edwards, E. Fu, K. Helton, K. Nelson, M. R. Tam, and B. H Weigl, "Microfluidic diagnostic technologies for global public health," Nature, vol. 442, pp. 412-418, July 2006.

[10] S. Hiyama, Y. Moritani, T. Suda, R. Egashira, A. Enomoto, M. Moore, and T. Nakano, "Molecular communication," in Proc. of 2005 NSTI Nanotechnology Conference, pp. 391-394, 2005.

[11] T. Nakano, T. Suda, T. Koujin, T. Haraguchi, and Y. Hiraoka, "Molecular communication through gap junction channels," Springer Transactions on Computational Systems Biology X, vol. 5410, pp. 81-99, 2008.

[12] A. Enomoto, M. Moore, T. Nakano, R. Egashira, T. Suda, A. Kayasuga, H. Kojima, H. Sakakibara, and K. Oiwa, "A molecular communication system using a network of cytoskeletal filaments," in Proc. of 2006 NSTI Nanotechnology Conference, pp. 725-728, 2006.

[13] S. Hiyama, R. Gojo, T. Shima, S. Takeuchi, and K. Sutoh, "Biomolecular-motor-based nano- or microscale particle translocations on DNA microarrays," Nano Letters, vol. 9, no. 6, pp. 2407-2413, 2009.

[14] M. Gregori and I. Akyildiz, "A new nanonetwork architecture using flagellated bacteria and catalytic nanomotors," IEEE Journal on Selected Areas in Communications, vol. 28, no. 4, pp. 612-619, 2010.

[15] P. Lio and S. Balasubramaniam, "Opportunistic routing through conjugation in bacteria communication nanonetwork," Nano Communication Networks, vol. 3, pp. 36-45, Mar. 2012.

[16] N. Farsad, A. W. Eckford, S. Hiyama, and Y. Moritani, "Information rates of active propagation in microchannel molecular communication," in Proc. of 5th International ICST Conference on Bio-Inspired Models of Network, Information, and Computing Systems, (Boston, MA), 2010.

[17] N. Farsad, A. W. Eckford, S. Hiyama, and Y. Moritani, "A simple mathematical model for information rate of active transport molecular communication," in Proc. of 2011 IEEE INFOCOM Workshops, (Shanghai, P. R. China), pp. 473-478, 2011.

[18] N. Farsad, A. W. Eckford, and S. Hiyama, "Channel design and optimization of active transport molecular communication," in Proc. of 6th International ICST Conference on Bio-Inspired Models of Network, Information, and Computing Systems, (York, England), 2011.

[19] N. Farsad, A. W. Eckford, and S. Hiyama, "A mathematical channel optimization formula for active transport molecular communication," in Proc. of 2012 IEEE International Conference on Communications (ICC) Workshops, (Ottawa, Canada), 2012.

[20] A. W. Eckford, N. Farsad, S. Hiyama, and Y. Moritani, "Microchannel molecular communication with nanoscale carriers: Brownian motion versus active transport," in Proc. of 2010 IEEE International Conference on Nanotechnology, (Seoul, South Korea), pp. 854 - 858, 2010.

[21] T. Nitta, A. Tanahashi, M. Hirano, and H. Hess, "Simulating molecular shuttle movements: Towards computer-aided design of nanoscale transport systems," Lab on a Chip, vol. 6, no. 7, pp. 881-885, 2006. 